\title{
Drug Repurposing and New Therapeutic Strategies for SARS-CoV-2 Disease Using a Novel Molecular Modeling-AI Hybrid Workflow
}

\author{
Scott D. Bembenek \\ Denovicon Therapeutics, San Diego, CA, 92130, USA \\ Corresponding author: sbembenek@ denovicontx.com
}

\begin{abstract}
The recent outbreak of the novel coronavirus (SARS-CoV-2) poses a significant challenge to the scientific and medical communities to find immediate treatments. The usual process of identifying viable molecules and transforming them into a safe and effective drug takes 10-15 years, with around 5 years of that time spent in preclinical research and development alone. The fastest strategy is to identify existing drugs or late-stage clinical molecules (originally intended for other therapeutic targets) that already have some level of efficacy. To this end, we tasked our novel molecular modeling-AI hybrid computational platform with finding potential inhibitors of the SARS-CoV-2 main protease (M $\left.\mathrm{M}^{\text {pro }}, 3 \mathrm{CL}^{\mathrm{pro}}\right)$. Over 13,000 FDA-approved drugs and clinical candidates (represented by just under 30,000 protomers) were examined. This effort resulted in the identification of several promising molecules. Moreover, it provided insight into key chemical motifs surely to be beneficial in the design of future inhibitors. Finally, it facilitated a unique perspective into other potentially therapeutic targets and pathways for SARS-CoV-2.
\end{abstract}

Keywords: SARS-CoV-2, COVID-19, main protease, $\mathrm{M}^{\mathrm{pro}}, 3 \mathrm{CL}^{\text {pro }}$, 3-chymotrypsin-like protease, molecular modeling, computer-aided drug design, virtual screening, drug repurposing, artificial intelligence, AI, machine learning, mast cells, tryptase, PI3K, phosphoinositide 3-kinase 


\section{Introduction}

The novel coronavirus, SARS-CoV-2, continues to be a global health threat for which neither a treatment or vaccine currently exists. As of June 5, 2020, 6.74 million cases have been confirmed, resulting in 394,984 deaths globally. ${ }^{1}$

Bringing a drug to market takes some 10-15 years, with over 5 years of that spent in early research and development alone. Moreover, several thousand compounds will be made, roughly one or two will have the needed properties to move forward into clinical trials, and once there, the failure rate is $90-95 \%$. On the average, it takes 4 active projects just to deliver a single molecule to clinical trials, and a startling 57 projects are needed to achieve a marketed drug.

Given these challenges and the extended timeline, the most viable option for an 'immediate' therapeutic is to identify an existing drug or clinical candidate (originally made for another indication) that impacts the SAR-CoV-2. To this end, many automated molecular docking studies have been performed to find such molecules to potentially inhibit the SARS-CoV-2 main protease $\left(\mathrm{M}^{\text {pro }}, 3 \mathrm{CL}^{\mathrm{pro}}\right)$. And while molecular docking is a powerful tool for assessing proteinligand interactions and overall molecular conformation, docking scores rarely correlate with experimental binding affinities, and at best, allow one to reasonably group molecules based on rough score cutoffs.

In our current study, a set of some 13,000 FDA-approved drugs and clinical candidates (approximately 30,000 protomers) were interrogated for the main protease using automated molecular docking. However, we went beyond this initial effort by performing an extensive postdocking workflow that included: 1) docking pose refinement and minimization; 2) strain calculations; 3) RMSD calculations (initial versus refined pose); 4) another round of pose refinement using two different in-house methods; and 5) quantum mechanical calculations. In a separate analysis, these molecules were also interrogated by our artificial intelligence (AI) platform. In order to come away with a focused list of the most promising molecules, the best hits from each approach were compared. A given molecule was chosen if it hit in both tiers, or represented novel chemical matter for the main protease.

The result is five molecules (originally made for a diverse set of targets) that are of interest for their potential to engage the main protease, as novel chemical matter for future design efforts, and for the insight they give into other possible therapeutic targets and pathways.

\section{Methods}

\section{Platform Overview}

In general, at all stages of the drug discovery process (target selection, hit identification, hit to lead transformation, and lead optimization) the Denovicon computational platform uses a unique combination of ligand- and structure-based methods along with machine learning and artificial intelligence (AI) approaches. Here, only the hit identification workflow of the platform was implemented. Moreover, rather than virtual screening our proprietary databases for hits, we looked only at FDA-approved drugs and clinical candidates. 


\section{Ligand Preparation}

A database containing FDA-approved drugs and clinical candidates was created from several sources: The Binding Database FDA-approved Drugs ${ }^{2}$; ChEMBL Drugs ${ }^{3}$; Selleckchem FDAapproved Drug Library ${ }^{4}$; Enamine FDA-approved Drugs ${ }^{5}$ along with our own curation efforts, which resulted in over 13,000 molecules. Each molecule was prepared for modeling by accounting for all of the most probable tautomer and ionization states using a custom in-house workflow developed with Pipeline Pilot. ${ }^{6}$ Transformation to three-dimensional structures was accomplished through minimization using the MMFF94s forcefield. ${ }^{7}$ Where well-defined, stereochemistry was enforced and all resulting hits were further reassessed to insure proper configurations. This overall effort resulted in just under 30,000 molecules that were analyzed.

\section{Protein Analysis and Preparation}

We examined several crystal structures of the main protease (apo, ligand and fragment complexes) to assess potential-ligand interactions, key pharmacophore features, and sidechain flexibility. This information was then used at various stages during the hit identification workflow.

\section{Virtual Screening}

In the initial stage, molecular docking was performed using the main protease complexed with an N3 inhibitor (PDB ID 6LU7). ${ }^{8}$ To prepare the protein for docking, protons were added at physiological $\mathrm{pH}$, sidechains were corrected and alternate forms were considered. ${ }^{9}$ Finally, waters molecules and the ligand were removed, and the catalytic cysteine (C145) was restored to its original form prior to making the covalent bond. At this point, no active site residues were minimized. This was deliberate as it was determined that the current state of the active site residues were in an 'open' form optimal for the initial docking round.

\section{Post-Virtual Screening Refinement}

The initial docking was performed with rDock $^{10}$ using the exhaustive sampling approach (with the number of poses set to 100) and rigid protein. The active site was assigned using the N3 ligand for the ligand-placement method. To refine the number of hits down for more detailed analysis, the highest scoring pose (SCORE) for each molecule (with distinction for the protomer state) was retained. Finally, a 'generous' score (SCORE.INTER) cutoff of -25 was applied over all molecules. The overall effort resulted in over 7,000 molecules.

Starting from these best poses, each molecule was then placed back into the active site and minimized while now allowing key active site residues to relax with the final score determined using GBVI/WSA $\Delta \mathrm{G}$. With the minimized pose obtained, the binding strain and RMSD (versus the initial rDock pose) were calculated. Two different solvation models were considered (R-Field and Born) with the MMFF94s forcefield used throughout. ${ }^{9}$ Additionally, the two protonation states of the catalytic histidine (H41) were also assessed. The 7,000 molecules were then filtered down (using a combination of cutoffs for the strain, RMSD and the GBVI/WSA $\Delta \mathrm{G}$ score) to 
around 150 molecules. A subset of these molecules went through a more detailed pose analysis using two different in-house methods (one with a machine learning scoring function, the other with a forcefield-empirical hybrid scoring function) and quantum mechanical calculations.

\section{Artificial Intelligence (AI) Screening}

In addition to the molecular modeling tier, the 30,000 molecules were also interrogated with our AI platform, which has been trained on our curated database of biological small molecules, biological activities, therapeutic drug targets, therapeutic disease areas, and other key information. The resulting list of hits was compared to those (150 molecules) from the molecular modeling effort, and a final list was obtained.

Rather than generating an overwhelming list (of hundreds to thousands) of hits, our goal was to come away with a short, focused list of promising molecules. To this end, the best hits from each tier, molecular modeling and AI, were compared and priority for the final selection was given to molecules that: 1) hit in both tiers; or 2) represented novel chemical matter for the main protease.

\section{Results}

Here, we review the final set of molecules (Table 1.) resulting from the overall computational effort. The interactions with the main protease as determined from the molecular modeling workflow will be overviewed. In terms of the AI results, it is simple enough to assume that the compounds were found, once again, as viable main protease inhibitors. However, since many factors go into the AI platform, we take the opportunity to assert other reasonable (and furtherreaching) hypotheses.

Enzastaurin (LY317615, DB102) is a potent inhibitor of protein kinase C $\beta$ originally developed as an antiangiogenic cancer therapy. ${ }^{11}$ It also inhibits the phosphoinositide 3-kinase (PI3K) pathway. ${ }^{12}$ The PI3K pathway has been implicated in chronic airway inflammation, asthma ${ }^{13}$ and COPD. ${ }^{14}$ Moreover, the PI3K pathway has been added to the growing list of signaling factors and pathways that are activated by a variety of viruses as they depend on it for their replication process ${ }^{15-20}$. Indeed, an appropriately chosen PI3K inhibitor (most likely inhibiting the $\delta$ and/or $\gamma$ isoforms) in itself may have positive impact on the novel coronavirus and/or the resulting chronic lung inflammation of the disease (and we assume this was a trigger for the AI tier in identifying it).

The docking pose of Enzastaurin is shown in Figure 1. The upper portion of the dihydropyrroledione (kinase hinge) motif interacts with two oxyanion hole residues: S144 and C145 (G143 is the remaining oxyanion hole residue), while the bottom carbonyl interacts with H163. A series of $\pi$-proton interactions occur: two between the distal indole and N142; and one between the indole core and the backbone amine of E166. The core also benefits from the hydrophobic pocket formed by H41, M49, and M165. The (positively charged) piperidine also interacts with E166 via the backbone carbonyl to make a strong hydrogen bond. The distal pyridyl makes good hydrophobic interactions in a pocket formed by P168, Q189, and T190. Other hits that shared the key dihydropyrrole-dione motif, had a similar binding pose and overall similarity to Enzastaurin were found; in particular, (Pubchem ID) 448642 and 2403. Although the distal indole is shown 
rotated 'outwards' towards the back pocket, an alternate binding pose where it is rotated 'inwards' towards the core is anticipated as well; we have estimated the energy barrier to be approximately $1.6 \mathrm{kcal} / \mathrm{mol}$ in favor of the (shown) outward conformation.

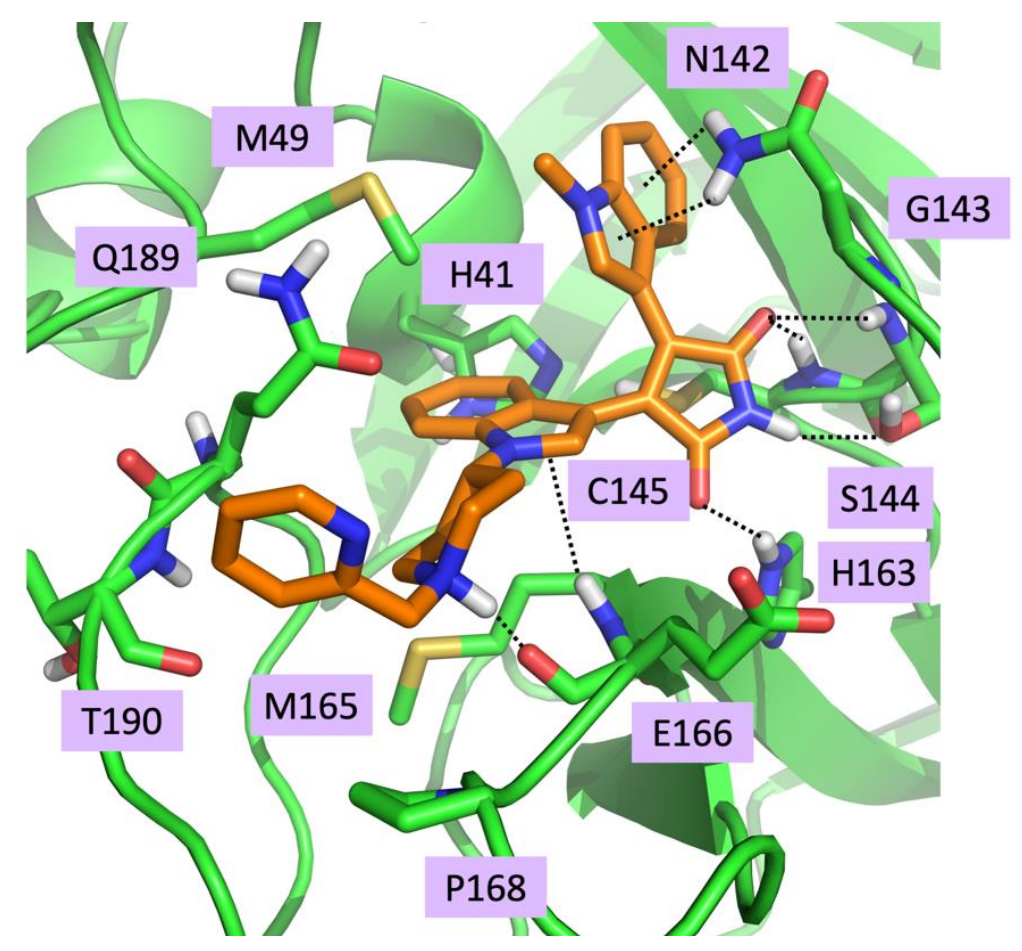

Figure 1. The proposed binding pose of Enzastaurin (LY317615, DB102) in the SARS-CoV-2 main protease. Specific (non-hydrophobic) interactions are shown with dashed lines. The distal indole (interacting with G143) is shown in an 'outward' conformation rotated towards the back pocket. Nonetheless, an 'inward' conformation where it is rotated towards the core was also found to be viable.

In 2019, the novel Respiratory Syncytial Virus (RSV) fusion (F) protein inhibitor Ziresovir (AK0529) completed a Phase 2 proof-of-concept study. For the first time ever, an antiviral agent proved successful in the treatment of infants hospitalized with RSV infection. ${ }^{21}$ Ziresovir demonstrated a dose-dependent clinical efficacy, whereby it clearly reduced patients' symptoms and viral loads. The study also confirmed the excellent safety profile seen in Phase 1. (It has completed two Phase 1 in healthy adult volunteers in Australia and China respectively, and one Phase 1 human mass balance study in the United Kingdom, ${ }^{22}$ along with multiple other clinical studies) We have not attempted to fully deconvolute the AI rational for the retrieval of this molecule, nonetheless, it is reasonable to assert that - in part - it honed in on the 'respiratory viral infection overlap' and perhaps certain similarities between proteins of RSV and SARSCoV-2.

The docking pose of Ziresovir in the main protease is shown in Figure 2. Within the oxyanion hole region, it makes a single $\pi$-proton interaction with G143. From the static docking pose, the sulfone does not appear to make direct interactions within the oxyanion hole. Nonetheless, we 
anticipate that at physiologic conditions where dynamics and waters will undoubtedly play a substantial role, the sulfone will make a series of direct and/or water-mediated interactions in the oxyanion hole region as well as with H163 and E166. The positively charge portion of the core makes a strong interaction with the carbonyl of Q189, while the terminal amine interacts with the backbone of T190. As did Enzastaurin, the core gains interactions made in the hydrophobic pocket formed by H41, M49, and M165. We accounted for nine possible protomers states of Ziresovir, and concluded that the one shown is the most viable along with that where the terminal amine is protonated, which would further enhance its interaction with T190.

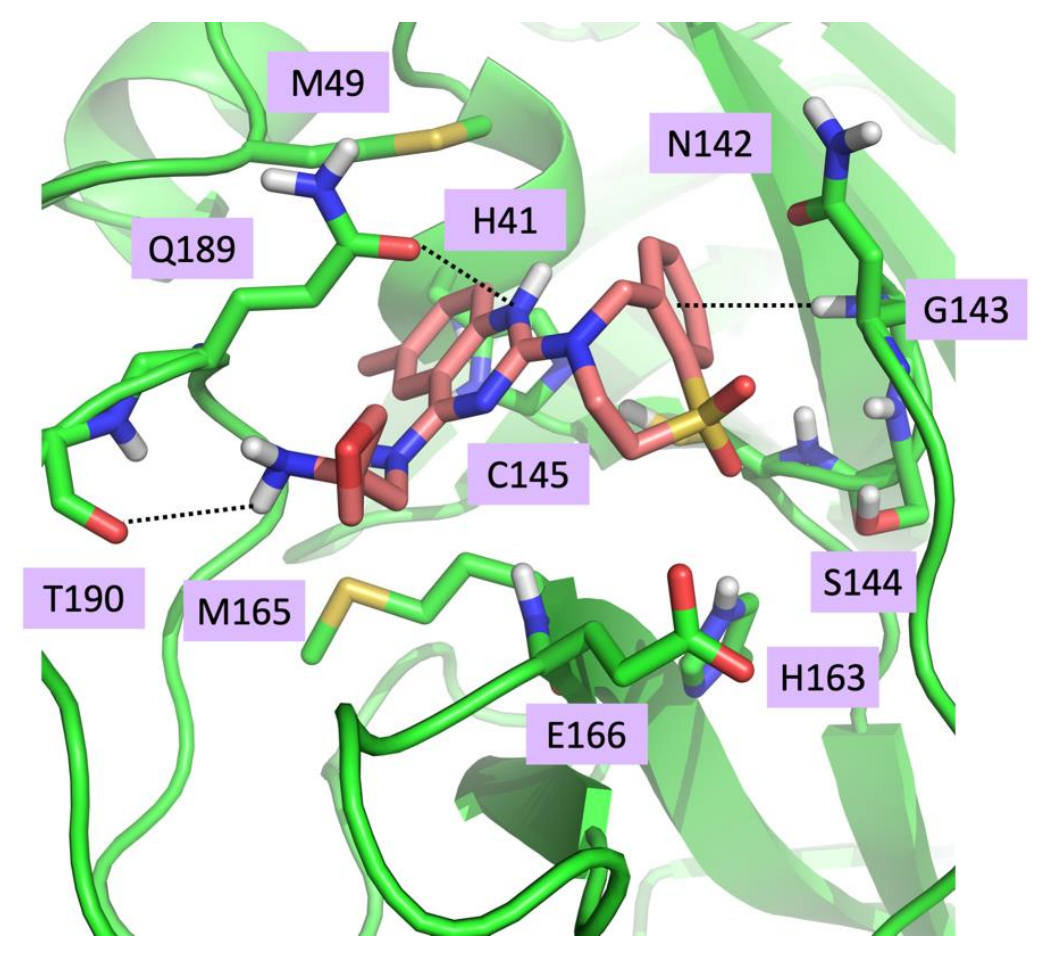

Figure 2. The proposed binding pose of Ziresovir (AK0529) in the SARS-CoV-2 main protease. Specific (non-hydrophobic) interactions are shown with dashed lines. Although the sulfone does not make direct interactions with the oxyanion hole, this region is solvent accessible and therefore, it is likely that water molecules may facilitate bridging interactions between the sulfone, the oxyanion hole residues, H163, and E166.

Razupenem (PTZ601, PZ-601, SMP-601 or SM-216601) belongs to the carbapenem subgroup of $\beta$-lactam antibiotics. It completed a Phase 2 study for the treatment of complicated skin and skin structure infections, but its progress has since halted due to high rates of rash adverse events. ${ }^{23}$ Nonetheless, Lenapenem and Ertapenem (marketed as Invanz) were also notable from our study, thus warranting an overall closer look at this antibiotic family (especially the orally available Tebipenem, which has been approved in Japan).

Figure 3 shows the proposed binding mode of Razupenem. The most striking interaction occurs between the carbapenem moiety and residues in the oxyanion hole, along with N142 and H163. Other interactions occur with Q189 between the core and terminal amine portions, where $\pi$ proton and charge-dipole interactions are made respectively. 
We note that electrophilic carbon of the carbapenem moiety is within striking distance of the catalytic cysteine (to within error of the predicted binding pose), which may allow for a covalent bond to be formed, thus endowing Razupenem (and possibly other carbapenem analogs) with improved 'affinity.' (The original docking efforts would not have explicitly accounted for this possibility, nor have we done the follow-up calculation yet). Indeed, a similar reaction occurs between certain $\beta$-lactam antibiotics and bacterial $\beta$-lactamases (where serine replaces the cysteine) resulting in bacterial resistance. To be sure, Razupenem and the other mentioned carbapenems were found exclusively from the molecular modeling effort and were not returned by the AI tier. Moreover, we note that $\beta$-lactam antibiotics such as amoxicillin and the penicillins did not score well.

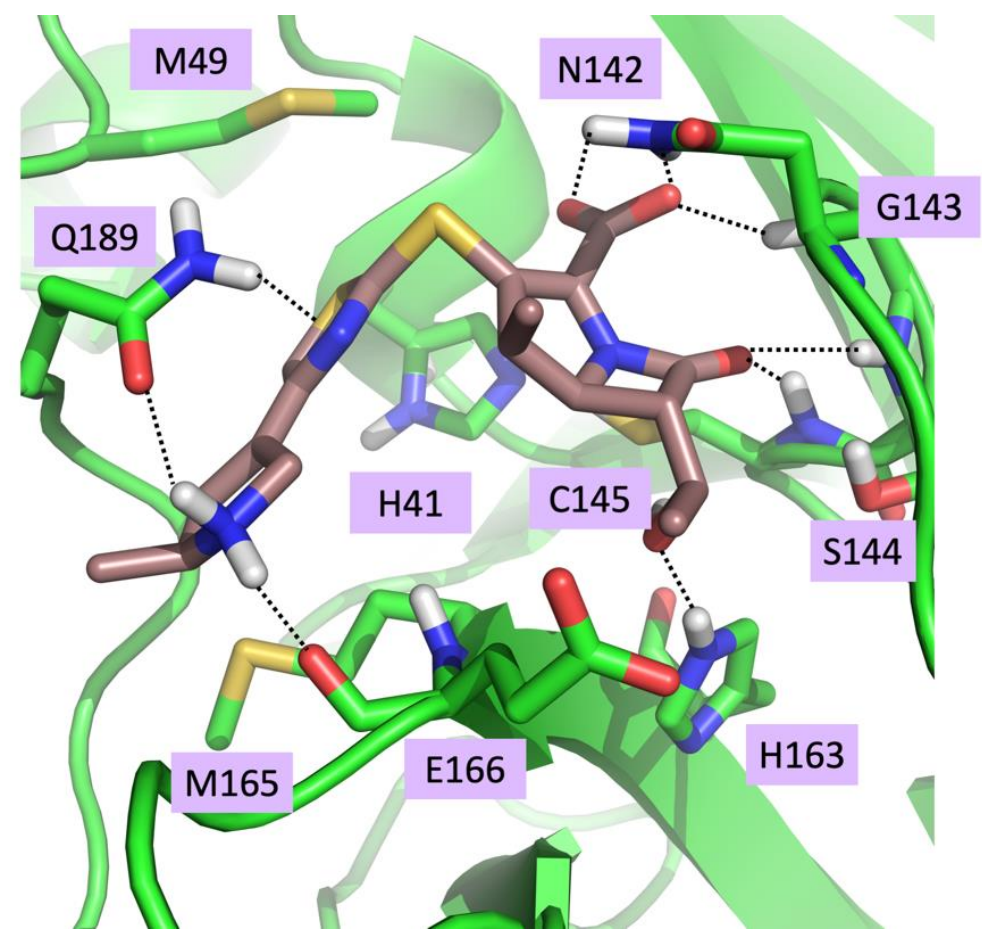

Figure 3. The proposed binding pose of Razupenem (PTZ601, PZ-601, SMP-601 or SM216601 ) in the SARS-CoV-2 main protease. Specific (non-hydrophobic) interactions are shown with dashed lines.

Like Enzastaurin, Nemiralisib (GSK-2269557) inhibits the PI3K-dependent pathway, specifically, through phosphoinositide 3-kinase $\delta$ (PI3K $\delta$ ). It was being developed for the treatment of inflammatory airways disease such as chronic adult asthma and chronic obstructive pulmonary disease (COPD), but more recently ${ }^{24}$ activated PI3K $\delta$ syndrome has become the main focus. As it did for Enzastaurin, all these factor were probably targeted by the AI.

In Figure 4 the key interactions are as follows: a hydrogen bond between the nitrogen of the oxazole motif and G143; two hydrogen bonds are made with the indazole motif, one with S144 and another with E166, while a $\pi$-cationic interaction is made with $\mathrm{H} 163$; an electrostatic 
interaction between the terminal indole and the carbonyl backbone of $\mathrm{H} 41$ (the orientation is a bit off to be a true hydrogen bond); and a strong hydrogen bond between the protonated nitrogen of the piperazine and T25. The molecule also benefits from the hydrophobic pocket created by L27, H41, C145, and M165.

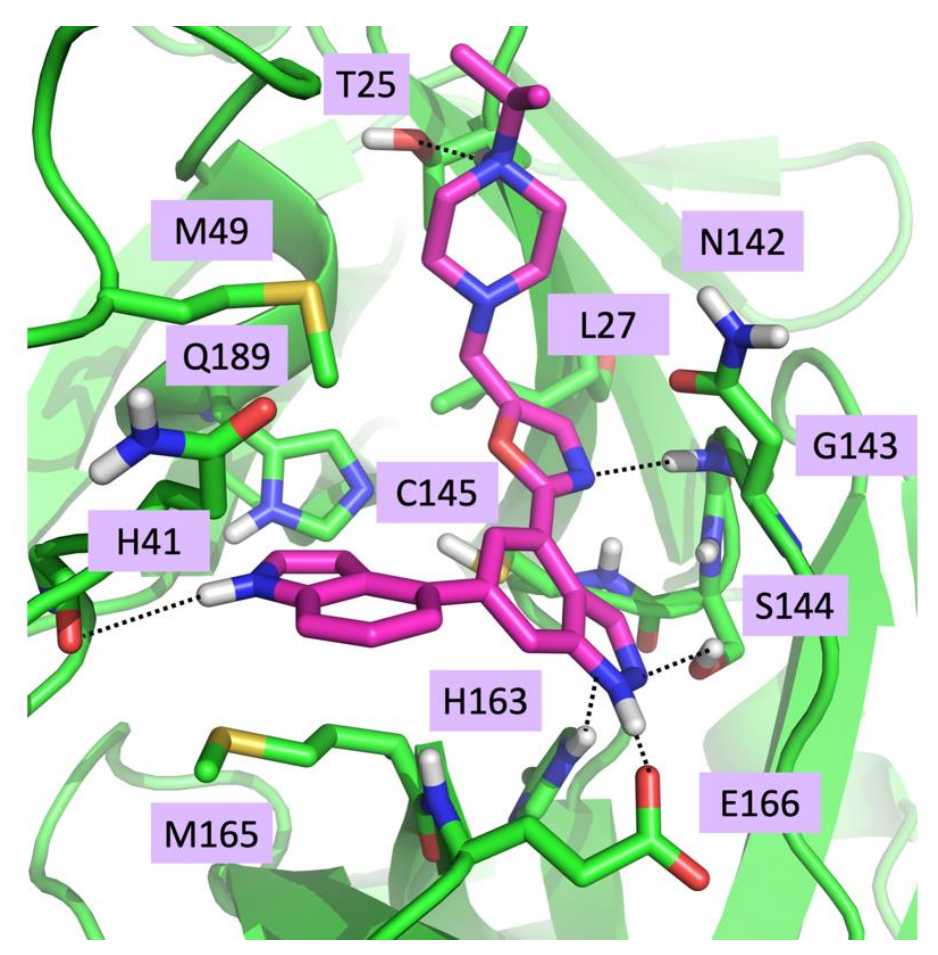

Figure 4. The proposed binding pose of Nemiralisib (GSK-2269557) in the SARS-CoV-2 main protease. Specific (non-hydrophobic) interactions are shown with dashed lines.

RWJ-56423 is a selective human mast cell tryptase inhibitor. ${ }^{25}$ The importance of mast cells in allergic disease is well established. Moreover, they play an essential role in protecting the host against certain pathogens (such as viruses). Nonetheless, these interactions are complex and can contribute to the pathology of allergic disease, result in inappropriate inflammatory response and outright detrimental effects. ${ }^{26-28}$ In general, the goal of a tryptase inhibitor is to mediate these types of negative responses. Indeed, in addition to any inhibitory effect it may have on the main protease inhibitor, its tryptase inhibiting effects may be beneficial as well and is most likely one of the reasons it showed up in the AI tier (the other reason may be because the main protease and the tryptase share some common structure as they are both proteases - cysteine and serine respectively). Interestingly, we note that dysfunctional mast cell activation and histamine release have recently been implicated in SARS-CoV-2 disease, thus owing to the possible therapeutic effect imparted by Famotidine. ${ }^{29}$

As seen in Figure 5, RWJ-56423 makes several key interactions as follows: two hydrogen bonds are made with the hydroxyl, one with N142 (via the carbonyl), one with G143; the amide carbonyl makes a hydrogen bond with H163; the other carbonyl makes a hydrogen bond with the amine backbone of E166; and the guanidine fits in an electronegative pocket formed by Y54 and 
D187. The guanidine also make a good $\pi$-cationic interaction with $\mathrm{H} 41$, and a hydrogen bond interaction with the backbone carbonyl of H164. We note that the putative electrophilic carbon for the covalent interaction in the tryptase ${ }^{25}$ is not proximal to the catalytic cysteine of the main protease (although it is proximal to the catalytic serine of the tryptase).

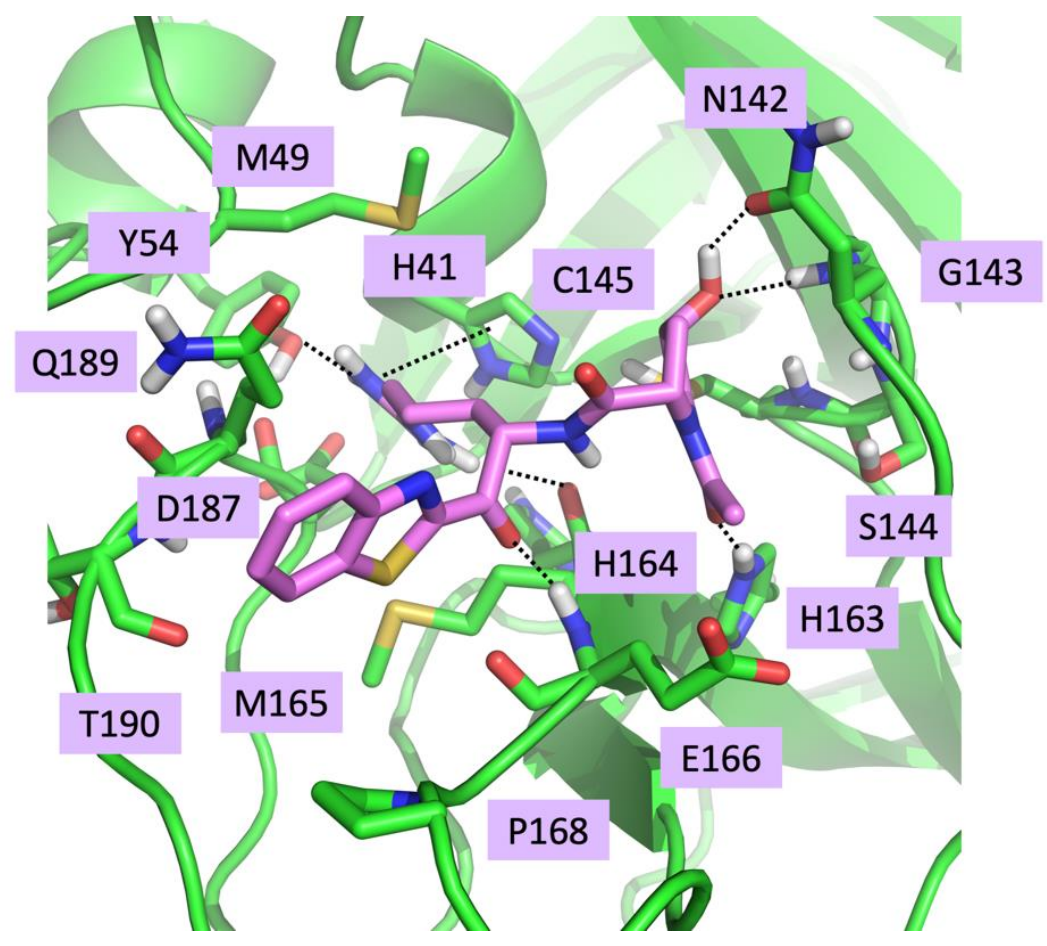

Figure 5. The proposed binding pose of RWJ-56423 in the SARS-CoV-2 main protease. Specific (non-hydrophobic) interactions are shown with dashed lines. 


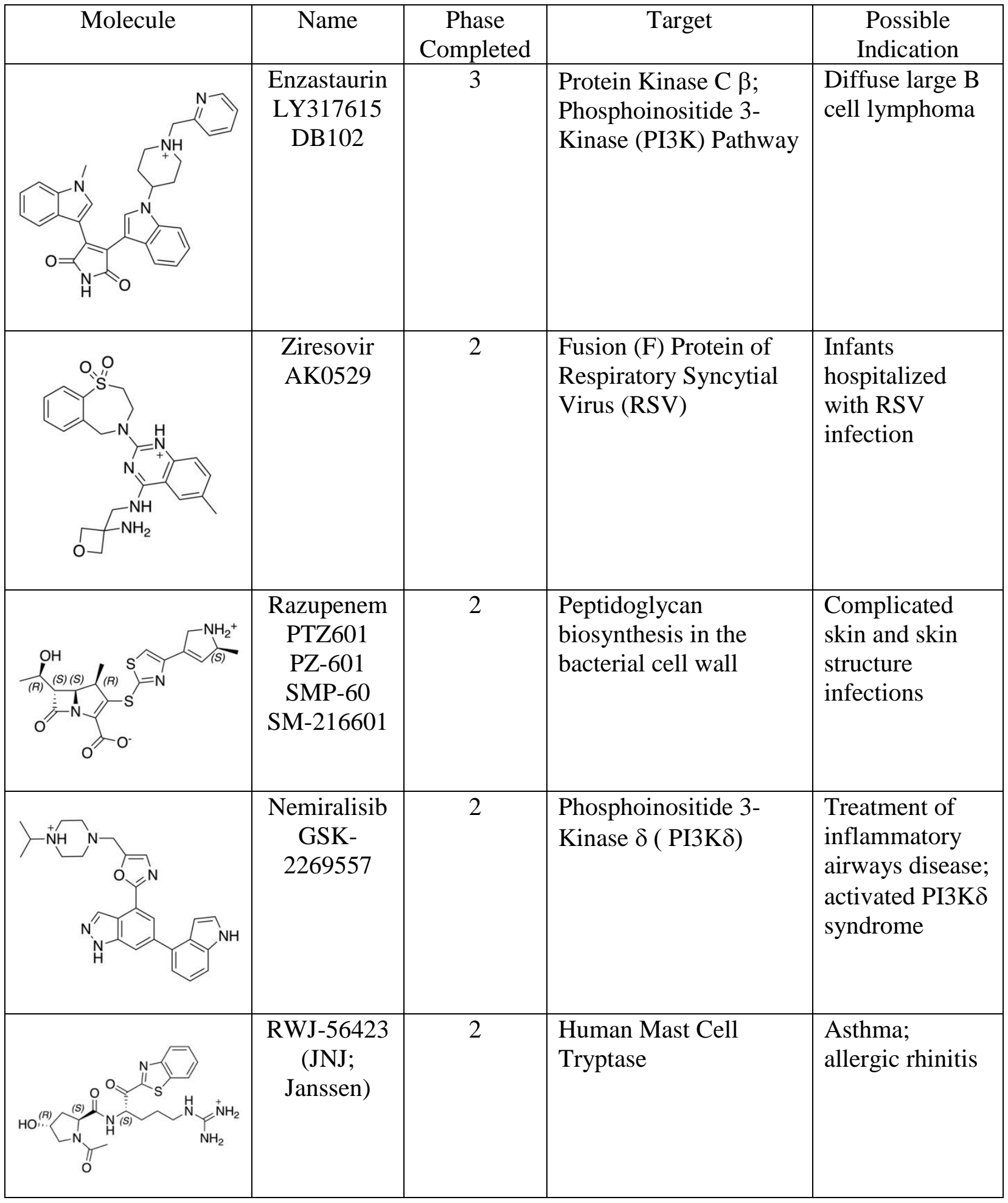

Table 1. The most viable hits (including their protomer state) as generated from the Denovicon virtual screening and AI platform. 


\section{Discussion}

Using a combination of molecular modeling and AI, we examined over 13,000 FDA-approved drugs and clinical candidates (approximately 30,000 protomers) for their potential efficacy against SARS-CoV-2 disease. The molecular modeling effort consisted of a virtual screening and a thorough post-virtual screening refinement workflow that ultimately led to 150 compounds of interest for the main protease. The 30,000 protomers were also examined using our AI workflow which, unlike the molecular modeling tier, was not restricted to inhibitors of the main protease. By keeping only those molecules that were the best hits in both tiers, or represented novel chemical matter for the main protease, we arrive at the five compounds shown in Table 1.

The five molecules represent a diverse set of structures originally made to engage a variety of targets for various indications (Ziresovir safety in infants makes it especially noteworthy). Through the molecular modeling they are connected as being potential inhibitors of the main protease, and while this may also be the implied connection gleaned from the AI, the rational is not known for sure; as such, we assert some reasonable hypotheses based on their original targets and indications.

In particular, efficacy against SARS-CoV-2 may result from the use of certain: 1) PI3K inhibitors; and/or 2) tryptase inhibits of human mast cells. Indeed, the role of the PI3K pathway in the replication process of certain virus is well established. ${ }^{15-20}$ Moreover, its role in inflammation in such conditions as COPD and asthma is also well known. Mast cells play a substantial role in certain allergic responses, as well as providing protection against certain pathogens, and overactive inflammatory responses with detrimental effects are known. ${ }^{26-28}$ Recently, dysfunctional mast cell activation and histamine release have been implicated in SARS-CoV-2 disease, in connection with the possible therapeutic effect of Famotidine. ${ }^{29} \mathrm{~A}$ natural extension of these hypotheses is that multiple-acting inhibitors or regimens consisting of a combination of inhibitors (e.g., main protease inhibitor and PI3K inhibitor) may have unique benefits. Only with further experimental information will it be known for sure.

Finally, the interactions seen from the docking poses drew attention to well-known drug discovery motifs that may provide useful in the design of future inhibitors of the main protease. The carbapenem motif of Razupenem makes several interactions in the oxyanion hole and is positioned to make a covalent bond with the catalytic cysteine, something it is well known to do with $\beta$-lactamases. The kinase hinge motif (dihydropyrrole-dione) of Enzastaurin also made numerous interactions in the oxyanion hole, and it was also seen in other initial hits we found (e.g., Pubchem ID: 448642 and 2403). 


\title{
References
}

\author{
${ }^{1}$ https://coronavirus.jhu.edu/map.html \\ 2 https://www.bindingdb.org/bind/ByFDAdrugs.jsp \\ ${ }^{3}$ https://www.ebi.ac.uk/chembl/g/\#browse/drugs \\ ${ }^{4}$ https://www.selleckchem.com/screening/fda-approved-drug-library.html \\ ${ }^{5}$ https://www.enaminestore.com/platedsets/fda-approved \\ ${ }^{6}$ https://www.3dsbiovia.com/products/collaborative-science/biovia-pipeline-pilot/
}

${ }^{7}$ We implement a pipeline pilot workflow that made use of RDKitPerformMinimization.py from MayaChemTools: http://www.mayachemtools.org/

${ }^{8}$ Jin, Z.; Du, X.; Xu, Y.; Deng, Y.; Liu, M.; Zhao, Y.; Zhang, B.; Li, X.; Zhang, L.; Peng, C.; Duan, Y.; Yu, J.; Wang, L.; Yang, K.; Liu, F.; Jiang, R.; Yang, X.; You, T.; Liu, X.; Yang, X.; Bai, F.; Liu, H.; Liu, X.; Guddat, L. W., Xu, W.; Xiao, G.; Qin, C.; Shi, Z.; Jiang, H.; Rao, Z.; and Yang, H. Structure of Mpro from COVID-19 virus and discovery of its inhibitors. Nature 2020, https://doi.org/10.1038/s41586-020-2223-y

${ }^{9}$ Chemical Computing Group. Molecular Operating Environment (MOE) Structure Preparation. 2020.

${ }^{10}$ Ruiz-Carmona, S.; Alvarez-Garcia, D.; Foloppe, N.; Garmendia-Dova, A. B.; Juhos, S.; Schmidtke, P.; Barril, X.; Hubbard, R. E.; Morley, S. D. rDock: A fast, versatile and open source program for docking ligands to proteins and nucleic acids. PLoS Comput. Biol. 2014, 10, https://doi.org/10.1371/journal.pcbi.1003571

${ }^{11}$ Ma, Shuo; Rosen, S. T. Enzastaurin. Curr. Opin. Oncol. 2007, 19, 590-595.

${ }^{12}$ Gelardi, T; Caputo, R; Damiano, V; Daniele, G; Pepe, S; Ciardiello, F; Lahn, M; Bianco, R; Tortora, G. Enzastaurin inhibits tumours sensitive and resistant to anti-EGFR drugs. British Journal of Cancer 2008, 99, 473480.

${ }^{13}$ Jeong, J. S.; Kim, J. S.; Kim, S. R.; Lee, Y. C. Defining bronchial asthma with phosphoinositide 3-kinase delta activation: towards endotype-driven management. Int. J. Mol. Sci. 2019, 20, 3525-3542.

${ }^{14}$ Pirozzi, F.; Ren, K.; Murabito, A.; Ghigo, A. PI3K signaling in chronic obstructive pulmonary disease: mechanisms, targets, and therapy. Current Medicinal Chemistry 2019, 26, 2791-2800.

${ }^{15}$ Ehrhardt, C. From virus entry to release: the diverse functions of PI3K during RNA virus infections. Future Virol. 2011, 6, 1225-1239.

${ }^{16}$ Ayllon, J.; García-Sastre, A.; Hale, B. G. Influenza A viruses and PI3K: are there time, place and manner restrictions? Virulence 2012, 3, 411-414.

${ }^{17}$ Diehl, N.; Schaal, H. Make yourself at home: viral hijacking of the PI3K/Akt signaling pathway. Viruses. 2013, 5, 3192-3212.

${ }^{18}$ Kindrachuk, J.; Ork, B.; Hart, B. J.; Mazur, S.; Holbrook, M. R.; Frieman, M. B.; Traynor, D.; Johnson, R. F.; Dyall, J.; Kuhn, J. H.; Olinger, G. G.; Hensley, L. E.; Jahrlinga, P. B. Antiviral potential of ERK/MAPK and $\mathrm{PI} 3 \mathrm{~K} / \mathrm{AKT} / \mathrm{mTOR}$ signaling modulation for middle east respiratory syndrome coronavirus infection as identified by temporal kinome analysis. Antimicrob Agents Chemother. 2015, 59, 1088-1099. 
${ }^{19}$ Wang, L.; Yang, L.; Fikrig, E.; Wang, P. An essential role of PI3K in the control of West Nile virus infection. Sci. Rep. 2017, 7, 3724-3732.

${ }^{20}$ Ranadheera , C.; Coombs, K. M.; Kobasa, D. Comprehending a killer: the Akt/mTOR signaling pathways are temporally high-jacked by the highly pathogenic 1918 influenza virus. EBioMedicine 2018, 32, 142-163.

${ }^{21}$ Beigel, J. H.; Nam, H. H.; Adams, P. L.; Krafft, A.; Ince, W. L.; El-Kamary, S. S.; Sims, A. C. Advances in respiratory virus therapeutics - A meeting report from the 6th isirv antiviral group conference. Antivir. Res. 2019, $167,45-67$.

${ }^{22}$ Zheng, X.; Gao, L.; Wang, L.; Liang, C.; Wang, B.; Liu, Y.; Feng, S.; Zhang, B.; Zhou, M.; Yu, X.; Xiang, K.; Chen, L.; Guo, T.; Shen, H. C.; Zou, G.; Wu , J. Z.; Yun, H. Discovery of Ziresovir as a Potent, Selective, and Orally Bioavailable Respiratory Syncytial Virus Fusion Protein Inhibitor. J. Med. Chem. 2019, 62, 6003-6014.

${ }^{23}$ Koulenti, D.; Xu, E.; Song, A.; Mok, I. Y. S.; Karageorgopoulos, D. E.; Armaganidis, A.; Tsiodras, S.; Lipman, J. Emerging treatment options for infections by multidrug-resistant gram-positive microorganisms. Microorganisms 2020, $8,191-230$

${ }^{24}$ Sanjeev Khindri; Anthony Cahn; Malcolm Begg; Mickael Montembault; Claudia Leemereise; Yi Cui; Annabel Hogg; Hannah Wajdner; Shuying Yang; Jon Robertson; J. Nicole Hamblin; Andrea Ludwig-Sengpiel; Oliver Kornmann; Edith M. Hessel. A multicentre, randomized, double-blind, placebo-controlled, crossover study to investigate the efficacy, safety, tolerability, and pharmacokinetics of repeat doses of inhaled nemiralisib in adults with persistent, uncontrolled asthma. J. Pharmaco.l Exp. Ther. 2018, 367, 405-413.

${ }^{25}$ Costanzo, M. J.; Yabut, S. C.; Almond, Jr, H. R.; Andrade-Gordon, P.; Corcoran, T. W.; de Garavilla, L.; Kauffman, J. A.; Abraham, W. M.; Recacha, R.; Chattopadhyay, D.; Maryanoff, B. E. Potent, small-molecule inhibitors of human mast cell tryptase. antiasthmatic action of a dipeptide-based transition-state analogue containing a benzothiazole ketone. J. Med. Chem. 2003, 46, 3865-3876.

${ }^{26}$ Marshall, J. S. Mast-cell responses to pathogens. Nat. Rev. Immunol. 2004, 4, 787-799.

${ }^{27}$ Woldemeskel, M. A brief review of mast cells in microbial infection (inflammation) and tumor-associated angiogenesis. J. Clin. Exp. Pathol. 2015, 5, https://doi.org/10.4172/2161-0681.1000e119.

${ }^{28}$ Marshall, J. S.; Portales-Cervantes, L.; Leong, E. Mast cell responses to viruses and pathogen products. Int. J. Mol. Sci. 2019, 20, 4241-4258.

${ }^{29}$ Malone, R. W.; Tisdall, P.; Fremont-Smith, P.; Liu, Y.; Huang, X.; White, K. M.; Miorin, L.; Moreno Del Olmo, E.; Alon, A.; Delaforge, E.; Hennecker, C. D..; Wang, G.; Pottel, J.; Bona, R.; Smith, N.; Hall, J. M.; Shapiro, G.; Clark, H.; Mittermaier, A.; Kruse, A. C.; García-Sastre, A.; Roth, B. L.; Glasspool-Malone, J.; Francone, V.; Hertzog, N.; Fremont-Smith, M.; Ricke, D. O. COVID-19: Famotidine, histamine, mast cells, and mechanisms. Research Square https://doi.org/10.21203/rs.3.rs-30934/v1. 